\title{
Color Reproduction Using Riemann Normal Coordinates
}

\author{
Satoshi Ohshima ${ }^{1}$, Rika Mochizuki ${ }^{1}$, Jinhui Chao ${ }^{1}$, and Reiner Lenz ${ }^{2}$ \\ 1 Graduate School of Science and Engineering \\ Chuo University \\ 1-13-27, Kasuga, Bunkyo-ku, Tokyo Japan \\ jchao@ise.chuo-u.ac.jp \\ 2 Department Science and Technology \\ Linköping University \\ SE-60174 Norrköping, Sweden \\ reile@itn.liu.se, \\ http://www.itn.liu.se/ ${ }^{\sim}$ reile
}

\begin{abstract}
In this paper we use tools from Riemann geometry to construct color reproduction methods that take into account the varying color perception properties of observers. We summarize the different steps in the processing: the estimation of the discrimination thresholds, the estimation of the Riemann metric, the construction of the metricpreserving or color-difference-preserving mapping and the usage of Semantic Differentiation (SD) techniques in the evaluation. We tested the method by measuring the discrimination data for 45 observers. We illustrate the geometric properties of the color spaces obtained and show the result of the metric-preserving maps between the color space of colornormal observers and a color-weak observer. We then apply the obtained maps to produce color corrected reproductions of two natural images.
\end{abstract}

\section{Introduction}

All color reproduction methods in use today are rather rigid in the sense that the goal of the reproduction is decided in advance and often based on some kind of standard. A typical example is a print where the tolerance of the final product is specified in terms of some $\Delta E$. This does not take into account that color perception varies from observer to observer and even changes depending on many other factors. This approach is sufficient for most cases since human perception is quite flexible and reasonably similar between different observers. An extreme case where it obviously fails is color blindness where the observer is unable to see the difference between different pairs of colors. A color reproduction strategy that aims at reproduction for individual observers can only be realized in a feedback loop where the reproduction process takes into account the color perception properties of the intended observer. In computer vision a similar strategy is known as "active vision". An active vision system interacts with its environment by changing camera parameters or other imaging modalities to collect as much information as possible while analyzing the scene.

A. Trémeau, R. Schettini, and S. Tominaga (Eds.): CCIW 2009, LNCS 5646, pp. 140 149, 2009.

(C) Springer-Verlag Berlin Heidelberg 2009 
In an adaptive reproduction system we can also try to interact with the user in order to create a reproduction that is based on the perception of the individual user. Instead of minimization of reproduction error in the working color spaces of output devices, a perception based color reproduction requires a colordifference-preserving map between the perceptional color spaces of observers. The fundamental problem with this approach is of course the difficulty to obtain a quantitative description of the perception properties of an observer. A standard method to characterize color perception properties is the measurement of discrimination thresholds that describe the local sensitivities of the observer. In this paper we describe some of our experiments where we characterize the color perception of color-normal and color-weak observers by discrimination thresholds. We then use Riemann normal coordinates 3 to build a descriptor of the color perception properties of an observer from the measured discrimination thresholds. In the third processing stage the Riemann normal coordinates are used to establish color-difference-preserving mappings between the color spaces of the observers. An evaluation of the effect of such mappings is very difficult since it requires the measurement of the subjective impressions created by the color reproductions. We use semantic differentiation (SD) techniques to evaluate the performance of the proposed reproduction strategy. This SD evaluation of the reproduction shows that our method can create very similar impressions to color-weak observer as that of color-normals.

\section{Riemann Color Spaces}

The standard tool to characterize color perception are color differences. The most accessible and reliable measurements are small or local color differences. The most popular such measurements are the so-called just noticeable-difference (jnd) thresholds or the discrimination thresholds. They are obtained by measuring the minimal color-difference between a reference color and a test color that the observer can detect.

These threshold measurements provide at every reference color $\boldsymbol{x}_{o}$ a measure of local distances in color space as follows: use the reference color $\boldsymbol{x}_{o}$ as the origin and a color vector $\boldsymbol{x}=\boldsymbol{x}+\boldsymbol{\Delta}$ close to $\boldsymbol{x}_{o}$ as a test color. Then the discrimination ellipses/ellipsoids are points on the unit circles/spheres centered at the reference color $\boldsymbol{x}_{o}$. (Here the distance will be defined below). They are given by the color vectors $\boldsymbol{x}^{\prime}=\boldsymbol{x}_{o}+\boldsymbol{\Delta}^{\prime}$ where the increments $\Delta^{\prime}$ satisfy the equation:

$$
\left(\boldsymbol{\Delta}^{\prime}\right)^{T} \boldsymbol{G}\left(\boldsymbol{x}_{o}\right) \boldsymbol{\Delta}^{\prime}=1
$$

The positive definite matrix $\boldsymbol{G}\left(\boldsymbol{x}_{o}\right)$ is uniquely determined by the ellipses/ellipsoids and vice versa. With such a matrix $\boldsymbol{G}\left(\boldsymbol{x}_{o}\right)$ defined at every $\boldsymbol{x}_{o}$, the local distance around $\boldsymbol{x}_{o}$ can be computed as

$$
\left\|\boldsymbol{x}-\boldsymbol{x}_{o}\right\|^{2}=\|\boldsymbol{\Delta}\|^{2}=\boldsymbol{\Delta}^{T} \boldsymbol{G}\left(\boldsymbol{x}_{o}\right) \boldsymbol{\Delta}
$$

A space with a local distance defined by a matrix $\boldsymbol{G}(\boldsymbol{x})$ at every point $\boldsymbol{x}$ is called a Riemann space with Riemann metric $\boldsymbol{G}(\boldsymbol{x})$. The exact definition and technical details can be found in every book on Riemann geometry, for example in [2]. 
We estimate the matrices $\boldsymbol{G}(\boldsymbol{x})$ as follows: First we select a color vector $\boldsymbol{x}_{0}$ as a reference color. This vector is used as the origin of the ellipsoid. The observer varies a test color in a certain direction until he/she can see a difference to the reference color. Then this experiment is repeated but now the direction of the variation is modified. The result of $N$ such experiments is a sequence of $N$ color vectors $\boldsymbol{x}_{n}, n=1 \cdots N$ that are by definition located on the surface of the ellipsoid defining the just-noticeable differences.

In our experiments we measured the discrimination threshold ellipsoids using a 10 degree visual field of size $14 \mathrm{~cm} \times 14 \mathrm{~cm}$ seen from a distance of $80 \mathrm{~cm}$. The discrimination threshold data were measured for 45 college students (38 male, 7 female, 1 color-weak) in CIEXYZ coordinates. We choose ten uniformly distributed points within the gamut of the monitor as reference colors. We used 14 different directions for each reference color. The distribution of the 14 directions is not uniform but denser around the direction of the confusion lines and the long axes of threshold ellipsoids. The experimental setup is shown in Figure 1.

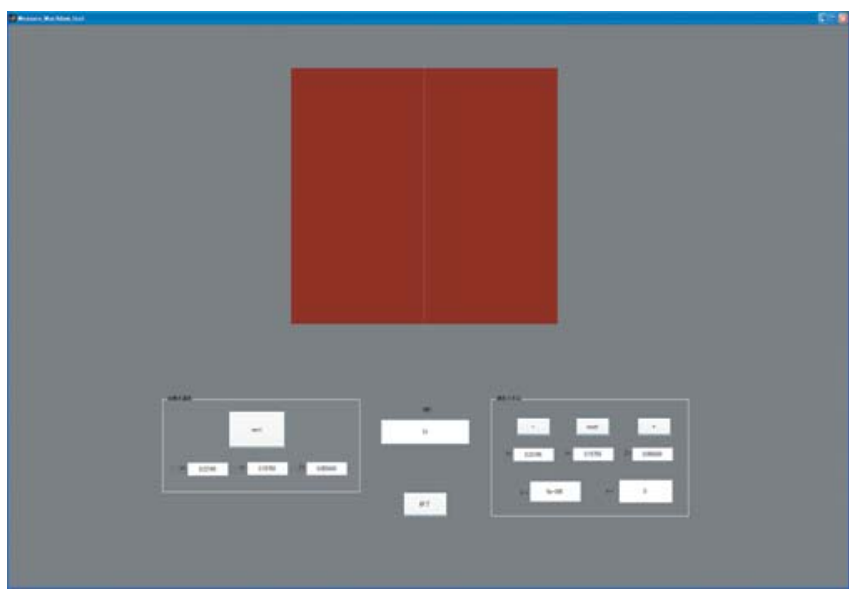

Fig. 1. Experimental setup

After the measurement of the jnd-vectors we have to compute the matrix $\boldsymbol{G}\left(\boldsymbol{x}_{o}\right)$ as the solution of the $N$ equations $1=\boldsymbol{\Delta}_{n}^{T} \boldsymbol{G}\left(\boldsymbol{x}_{o}\right) \boldsymbol{\Delta}_{n}, n=1, \cdots, N$ with $\boldsymbol{x}_{n}=\boldsymbol{x}_{o}+\boldsymbol{\Delta}_{n}$.

Writing $\boldsymbol{\Delta}=(x, y, z)$ and $\boldsymbol{G}=\left(\begin{array}{lll}a & b & c \\ b & d & e \\ c & e & f\end{array}\right)$ every measurement (represented by the vector $\boldsymbol{\Delta}$ ) gives one equation in the matrix entries $a$ to $f$ :

$$
a x^{2}+b y^{2}+c z^{2}+d x y+e x z+f y z=1
$$

The matrix entries $a$ to $f$ are obtained by least squares fitting (for the details see 5 ).

The characteristics of color-normal perception are represented by a matrix whose entries are the averages of the entries in the matrices of 44 color-normal 


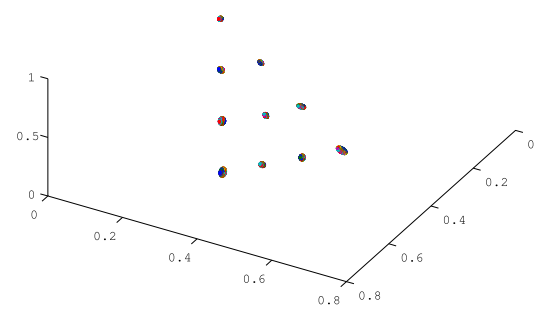

(a) Color-normals

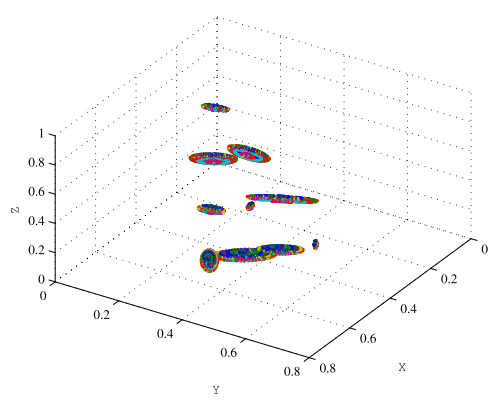

(b) Color-weak

Fig. 2. Discrimination threshold ellipsoids

observers. The average threshold ellipsoid of the color-normals and the thresholds of the color-weak observer are shown in Fig. 2a and Fig. 2b, The threshold ellipses of color-normal and the color weak are obtained as intersection between the above ellipsoids with the chromaticity plane, they are shown in Fig. 3a and Fig. $3 \mathrm{~b}$,

An even more important quantity in color perception is the difference between two colors with a larger color difference. They are however more subjective and therefore harder to deal with. In a Riemann color space the geometric distance between two color points $\boldsymbol{x}_{1}$ and $\boldsymbol{x}_{2}$ can be defined as the length of the shortest curve connecting the two points. Such shortest curves are known as geodesics. When the matrices $\boldsymbol{G}(\boldsymbol{x})$ are known for all points $\boldsymbol{x}$ then it is known that the geodesics are solutions to differential equations and they can be computed using numerical differential equation solvers. Using this approach in practice is however complicated by the fact that in this application the measurements of the thresholds is very time-consuming with the result that there are only very few samples available. Interpolation is therefore crucial in the estimation. In our implementation the interpolation uses the Akima algorithm [1].

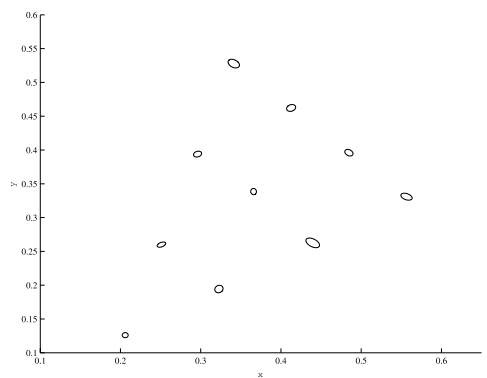

(a) Color-normals

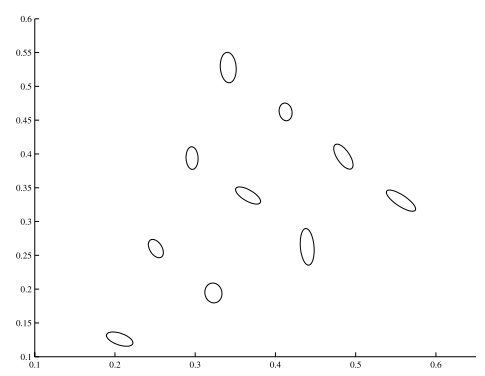

(b) Color-weak

Fig. 3. Discrimination threshold ellipses 


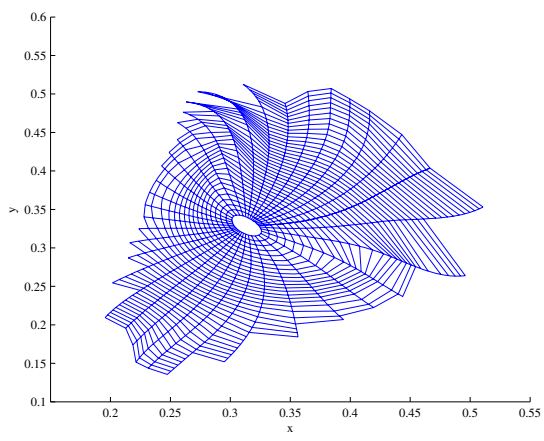

(a) Color-normals

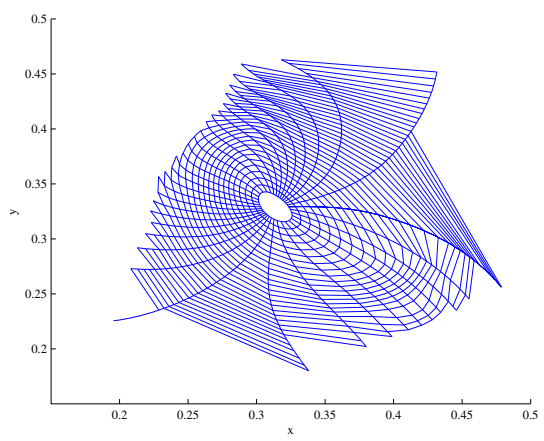

(b) Color-weak

Fig. 4. Riemann normal coordinates

On a Riemann color space we can use the geodesics and construct a coordinate system as follows: We first select a point of origin $\boldsymbol{x}_{o}$. Then we connect the points of the same distance from $\boldsymbol{x}_{o}$ on the neighboring geodesics. This gives a generalized polar coordinate system on the Riemann space similar to the coordinate system on the sphere with the north (or south) pole and the longitude/latitude coordinates. These coordinates are known as Riemann or normal coordinates.

In the following two figures the measured ellipsoids where first projected onto the chromaticity plane. Then the corresponding ellipses were computed and from them Riemann normal coordinates are obtained. In Figure 4a the solution computed from the color normal observers and in Figure 4b) the corresponding Riemann normal coordinates for the color weak observer are shown.

\section{Color Mapping Using Discrimination Threshold Matching}

In the previous sections, we described the construction of a Riemann color space from discrimination thresholds. As a result we obtain a geometric description of the color perception of an observer either locally in the form of a Riemann space with its metric or globally in the form of Riemann normal coordinates.

Given two Riemann color spaces we now construct a mapping between these color spaces that preserves the metric or color-difference properties of these spaces. We explain the basic idea by constructing an isometry between the color spaces of a color-weak observer and the color space of color-normal observers. We call such a map a "color weak" map (for a detailed description see [5]). 


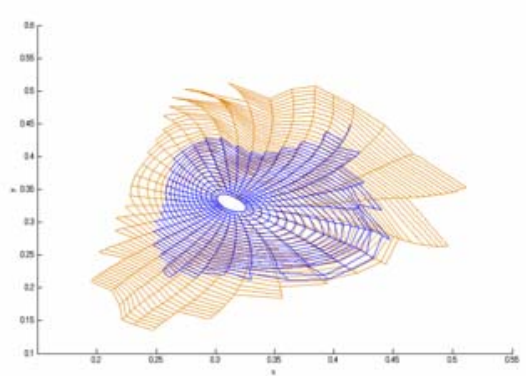

(a) Color-weak onto color-normal

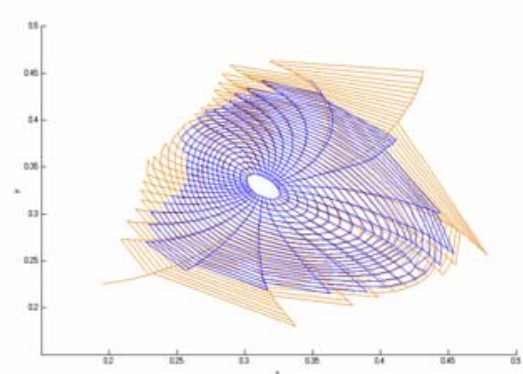

(b) Color-normal onto color-weak

Fig. 5. Images of geodesic coordinates

If the color space of the color weak observer is $C_{w}$ and the color space of the color normal observers is $C_{n}$ then the color-weak map is a function

$$
w: C_{w} \longrightarrow C_{n}, \quad \boldsymbol{x} \longmapsto \boldsymbol{y}=w(\boldsymbol{x})
$$

This mapping maps a color stimulus $\boldsymbol{x}$ perceived by color-normals to $\boldsymbol{y}=w(\boldsymbol{x})$ perceived by a color-weak observer (for details see [6] and [4). For the metric $\boldsymbol{G}_{n}(\boldsymbol{x})$ of the color-normal to be mapped isometrically to corresponding metric $\boldsymbol{G}_{w}(\boldsymbol{y})$ of the color-weak, the Jacobian matrix $\boldsymbol{D}_{w}$ of $w$ must fulfill the threshold matching condition:

$$
\boldsymbol{G}_{w}(\boldsymbol{y})=\left(\boldsymbol{D}_{w}\right)^{T} \boldsymbol{G}_{n}(\boldsymbol{x}) \boldsymbol{D}_{w}
$$

Applying $w$ to the input image and showing it to color-normal observers will provide them the same experience as the color-weak observer. Applying the inverse map $w^{-1}$ and presenting it to the color weak observer should give him the same impression as the original image to the color normal observer.

In order to evaluate the precision of the color-difference-preserving maps we calculated the image of Riemann normal coordinates of color spaces of colorweak and color-normals under the simulation map and the correction map. The results are plotted in the following figures.

The precision of the Riemann normal coordinates is determined by the numerical accuracy of interpolation algorithm and the ODE solver to draw geodesics. The upper limit of errors in the maps reading out from the Riemann normal coordinates is the size of the grid cell. Here we used the ODE solver based on the Runge-Kutta algorithm in Matlab.

In Fig. [5a, the blue coordinates show the image of geodesic coordinates of the color-weak under the simulation map, which is observed to be well fitted onto the yellow geodesic coordinates of the color-normal. Thus the color-differencepreservation of the color-weak or simulation map is confirmed. In Fig. 5b, the blue coordinates are the geodesics of the color-normal under the correction map 
or the inverse of the color-weak map. Also here we have a good fitting which means that the color-differences are preserved by the correction map.

\section{Color Correction of Natural Images}

The proposed correction algorithm is applied to natural images shown in Fig. 6b and Fig. 7b, The simulated color-weak images are in Fig. 6a and Fig. 7a, the corrected images for color-weak are in Fig. 6c and Fig. 7c respectively.

The intended application of the correction method is the production of an image that gives two different observers with different color vision properties the same impression. Since these effects are not measurable we cannot evaluate the quality of the methods quantitatively. Instead we use the standard Semantic Differential (SD) method to evaluate the impression of color-normals and color-weak. We prepared a questionnaire and let one color-normal and the colorweak observer judge ten polar pairs of most relevant adjectives with respect to the original images. A questionnaire with a seven level SD score is used in the evaluation.

Figures 8 and 9 show the comparisons of the SD scores between color-normals and the color-weak, before and after correction. The solid line shows the score of the color-normal and the dotted line that of the color-weak. In the SD evaluations shown on the left both color-normals and the color-weak see the original image before correction. The evaluations of the correction are shown on the right. Here the color-normal observer sees the original while the color-weak sees the corrected image.

The closeness of the SD curves after correction show that the color-normals and color-weak achieved the similar visual impression on the original image and the corrected image respectively.

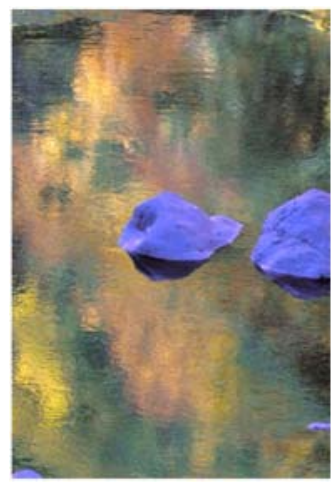

(a) Simulated colorweak image

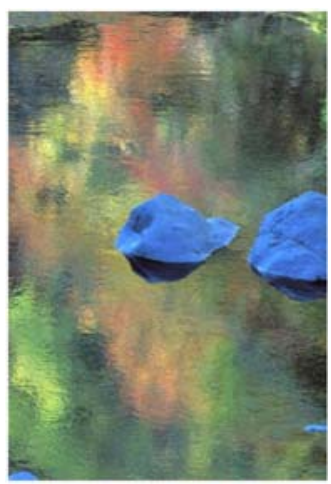

(b) Original

(c) Corrected image for the color-weak

Fig. 6. Rock Example 


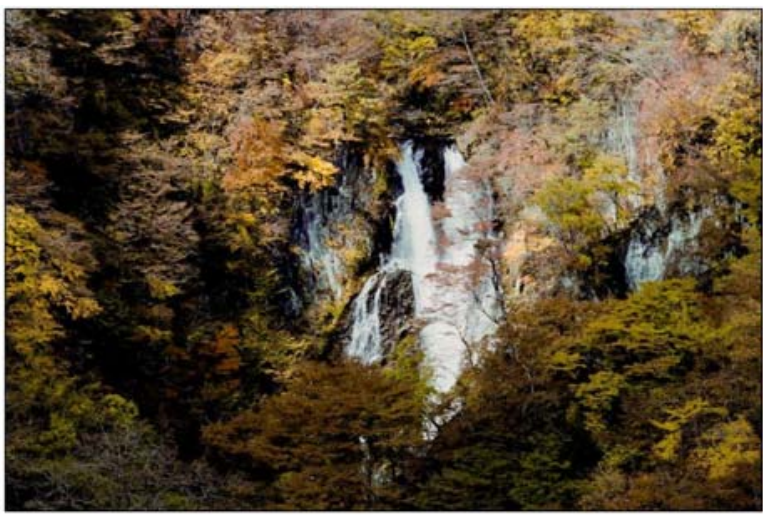

(a) Simulated color-weak image

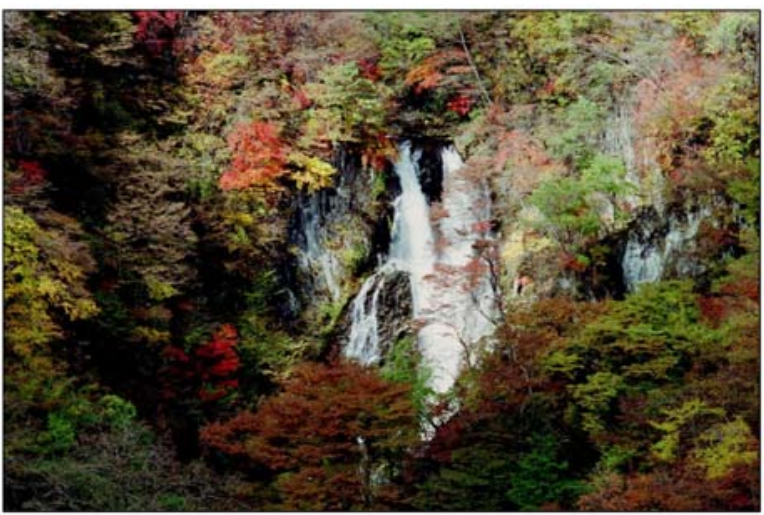

(b) Original

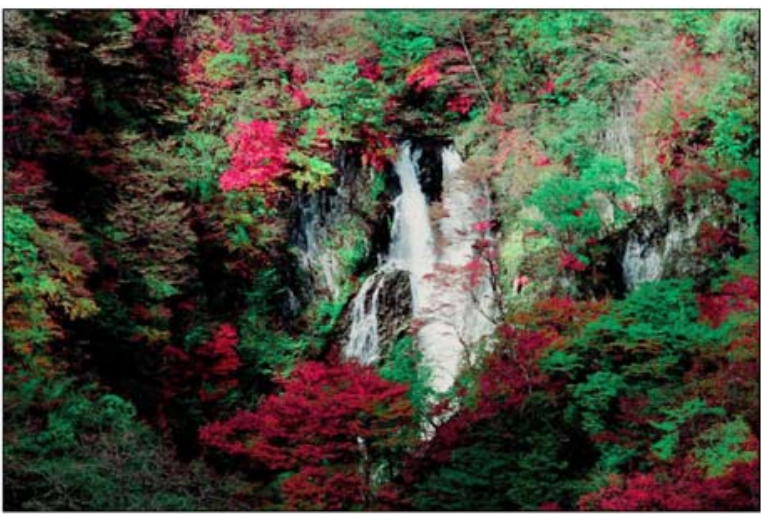

(c) Corrected image for the color-weak

Fig. 7. Fall Example 


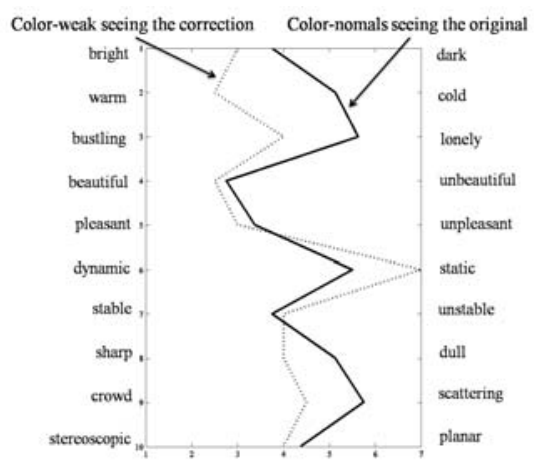

(a) SD evaluation of 6b before correction

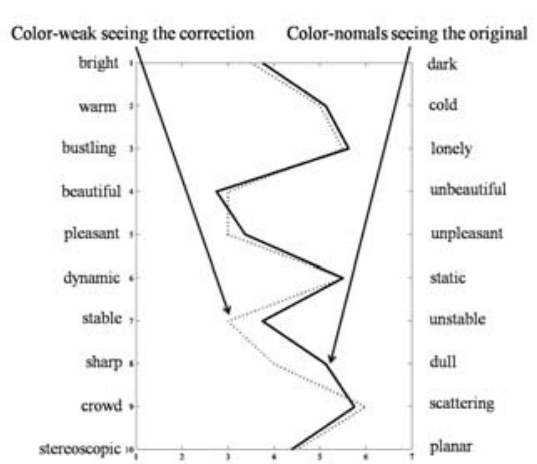

(b) SD evaluation of $6 \mathrm{~b}$ after correction

Fig. 8. SD evaluation of Fig. 6b before and after correction

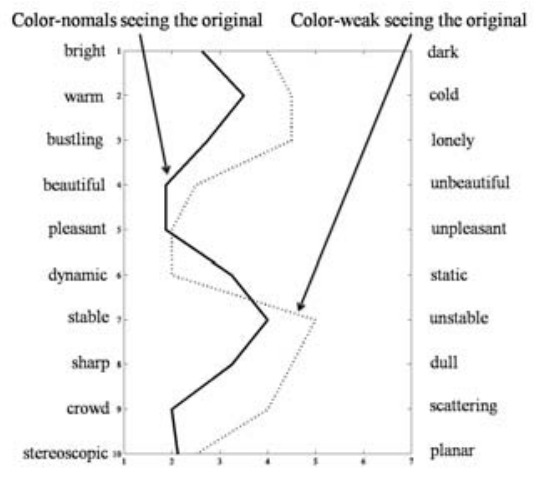

(a) SD evaluation of $7 \mathrm{~b}$ before correction

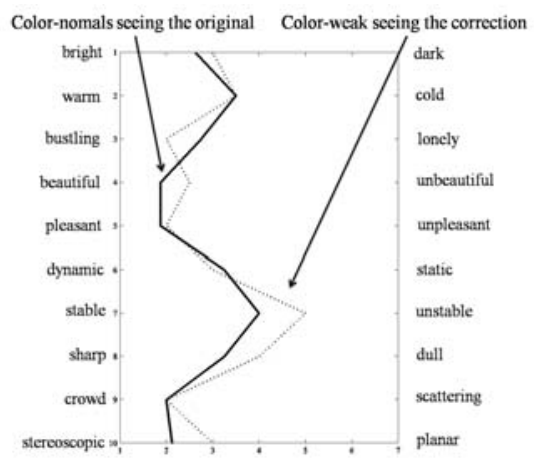

(b) SD evaluation of $7 \mathrm{~b}$ after correction

Fig. 9. SD evaluation of Fig. $7 \mathrm{~b}$ before and after correction

\section{Conclusion}

We described how the theory and tools of Riemann geometry can be used to construct maps that compensate the differences in the color perception of different observers. We demonstrated its application in a color correction for color weak vision and evaluated the resulting effects with the help of an SD-procedure.

\section{Acknowledgements}

This research is partially supported by the Institute of Science and Engineering, Chuo University.

The financial support of the Swedish Science Foundation is gratefully acknowledged. 


\section{References}

1. Akima, H.: A Method of Bivariate Interpolation and Smooth Surface Fitting for Irregulary Distributed Data Points. ACM Transactions on Mathematical Software 4(2), 148-159 (1978)

2. Boothby, W.M.: An Introduction to Differentiable Manifolds and Riemannian Geometry. Academic Press, New York (1955)

3. Chao, J., Lenz, R., Matsumoto, D., Nakamura, T.: Riemann geometry for color characterization and mapping. In: Proc. CGIV, pp. 277-282. IS\&T, Springfield (2008)

4. Chao, J., Osugi, I., Suzuki, M.: On definitions and construction of uniform color space. In: Proc. CGIV, pp. 55-60. IS\&T, Springfield (2004)

5. Mochizuki, R., Nakamura, T., Chao, J., Lenz, R.: Correction of color-weakness by matching of discrimination thresholds. In: Proc. CGIV, pp. 208-213. IS\&T, Springfield (2008)

6. Suzuki, M., Chao, J.H.: On construction of uniform color spaces. IEICE Trans. Fundamentals Elec. Comm. Comp. Sci. E85A(9), 2097-2106 (2002) 